\title{
Acupoint Stimulation for Fibromyalgia: A Systematic Review of Randomized Controlled Trials
}

\author{
Huijuan Cao, ${ }^{1}$ Xun Li, ${ }^{1}$ Mei Han, ${ }^{1}$ and Jianping Liu ${ }^{1,2}$ \\ ${ }^{1}$ Center for Evidence-Based Chinese Medicine, Beijing University of Chinese Medicine, 11 Bei San Huan Dong Lu, \\ Chaoyang District, Beijing 100029, China \\ ${ }^{2}$ NAFKAM, University of Tromso, Tromso, NO-9037, Norway
}

Correspondence should be addressed to Jianping Liu; jianping_l@hotmail.com

Received 21 August 2013; Accepted 7 November 2013

Academic Editor: Karen J. Sherman

Copyright (C) 2013 Huijuan Cao et al. This is an open access article distributed under the Creative Commons Attribution License, which permits unrestricted use, distribution, and reproduction in any medium, provided the original work is properly cited.

\begin{abstract}
Background. Acupoint stimulation is popular for treatment of fibromyalgia though there is lack of comprehensive evaluation of current clinical evidence for its effect and safety. Objective. To systematically review the beneficial effects and safety of acupoint stimulation for fibromyalgia. Methods. We searched six electronic databases for randomized trials on acupoint stimulation for treatment of fibromyalgia. Two authors extracted data and assessed the trial quality independently. RevMan 5.2 software was used for data analyses with effect estimate presented as (standard) mean difference and a 95\% confidence interval. We defined minimum, medium, and large SMD effect sizes as 0.3, 0.5, and 0.75. Results. 16 RCTs with 1081 participants were involved in this review. Only two trials were evaluated as low risk of bias. Meta-analysis showed that acupuncture alone or combined with cupping therapy was superior to conventional medications on reducing pain scores and/or the number of tender points. However, acupuncture showed no better than sham acupuncture on pain reduction. There was no serious adverse event reported to be related to acupoint stimulation. Conclusions. Acupoint stimulation appears to be effective in treating fibromyalgia compared with medications. However, further large, rigorously designed trials are warranted due to insufficient methodological rigor in the included trials.
\end{abstract}

\section{Background}

As nonspecific rheumatism, fibromyalgia (FM) is a disorder in which typical symptoms are chronic widespread muscularskeletal pain and stiffness accompanying with fatigue, anxiety, sleep disorder, and/or irritable bowel syndrome [1]. The well known diagnostic criterion for this disease was developed by the American College of Rheumatology (ACR) in 1990 [2], and the latest version of this diagnostic criterion was updated in 2009 [3]. The main purpose of treatment for FM is to alleviate the pain and improve the quality of life for FM patients [4].

Without the curative medications for the entire scope of symptoms and disabilities associated with FM [4], complementary therapies are commonly used by FM patients, such as acupuncture, herbal medicine, and massage. Systematic reviews were also conducted to summarize the clinical evidence of therapeutic effect of those complementary therapies in treating FM. Mayhew and Ernst [5] collected results from five randomized controlled trials in 2007 and demonstrated that due to the small sample size and low methodological quality of included trials, acupuncture could not be recommended for FM. Three years later, other two systematic reviews $[6,7]$ draw the similar conclusions with three more trials included. Recently, a Cochrane review [8] which is entitled as "Acupuncture for treating fibromyalgia" was published. With nine included trials, it concluded moderate level evidence that acupuncture had no better effect for pain relief compared with sham acupuncture, and there was low to moderate level that evidence showed that acupuncture was better than standard therapy or antidepressant for improving pain. However, all these four reviews only included English articles and mainly observed the comparison between acupuncture and sham acupuncture. In our previous review [9], 12 trials focusing on acupuncture were included, and the result showed that even though acupuncture showed no significant effect compared with sham acupuncture (MD $-0.55,95 \%$ CI -1.35 to $0.24, P=0.17, I^{2}=69 \%$ ) on pain reduction, there were significant effect of acupuncture on reducing 
the number of tender points (MD $-3.21,95 \% \mathrm{CI}-4.23$ to $\left.-2.11, P<0.00001, I^{2}=0 \%\right)$, and pain scores $(\mathrm{MD}$ $-1.78,95 \%$ CI -2.24 to $\left.-1.32, P<0.00001, I^{2}=0 \%\right)$ compared with conventional medications. We also found that, besides acupuncture, other point stimulation therapies, such as cupping therapy, were commonly used in clinics for treatment of FM.

Acupoint stimulation therapy includes acupuncture, cupping therapy (which involves applying suction by placing a vacuumized, usually by fire, cup or jar on points or affected body surfaces to induce local hyperemia or haemostasis), moxibustion (which involves the controlled burning of material, typically mugwort herb, at certain points or areas of the body surface), point injection (which involves injecting medication into an acupuncture point), point embedding (which involves embedding in the skin over the point with a small needle (s) or medicated catgut), or combination of two or more of those acupoint stimulation.

In traditional Chinese medicine (TCM) theory, stagnation of qi activity leads to the stasis of blood which causes pain [10]. Therefore, the potential mechanism of acupoint stimulation for FM is to regulate the qi and blood, combined with dispelling cold and removing damp. Though acupoint stimulation was popularly employed in treating FM, there is no systematic review that evaluates the clinical evidence of all types of acupoint stimulation. This systematic review aims to update the evidence from RCTs to evaluate the therapeutic effect and safety of different types of acupoint stimulation therapies for FM.

\section{Methods}

2.1. Inclusion Criteria. We included parallel-group RCTs of any kind of point stimulation therapies including acupuncture, cupping therapy, point injection, point catgut embedding, or moxibustion, compared with no treatment, placebo, or conventional medication in patients with FM. We also included combined therapy with acupoint stimulation and other interventions compared with other interventions in RCTs, or combined therapy of two kinds of point stimulation therapies compared with medication or other interventions. FM should be diagnosed according to recognized criteria. Primary outcome was change of pain intensity, and secondary outcomes included improvement of relevant symptoms, such as depression or quality of life and adverse events. There was no limitation on language and publication type.

2.2. Identification and Selection of Studies. We searched China Network Knowledge Infrastructure (CNKI) (19792013), Chinese Scientific Journal Database VIP (1989-2013), Wan Fang Database (1985-2013), Chinese Biomedicine (SinoMed) database (1978-2013), PubMed (1966-2013), and the Cochrane Library (Issue 5, 2013), and all the searches ended at May 2013. The search terms included "fibromyalgia," "fibrosis," "fibrositis," "myofascitis," or "myofibrositis" combined with "acupuncture," "electroacupuncture," "auricular therapy," "acupoint," “point embedding," "point injenction," "cupping," "moxibustion," or "meridian." Two authors (Huijuan
Cao and Mei Han) selected studies for eligibility and checked against the inclusion criteria independently.

2.3. Data Extraction and Quality Assessment. Two authors (Huijuan Cao and Mei Han) extracted the data from the included trials independently. Selection bias (random sequence generation and allocation concealment), performance bias (blinding of participants and personnel), detection bias (blinding of outcome assessment), attrition bias (incomplete outcome data), reporting bias (selective reporting), and other bias were assessed according to the criteria from the Cochrane Handbook for Systematic Reviews of Intervention [11]. There were three potential bias judgments: low risk, high risk, and unclear risk. A judgment of low risk was made when all the seven items met the criteria as "low risk," a judgment of high risk of bias was made when at least one of the seven items was assessed as "high risk."

2.4. Data Analysis. Data were summarized using risk ratio (RR) with 95\% confidence intervals (CI) for binary outcomes or mean difference $(\mathrm{MD}) /$ standard mean difference (SMD) with $95 \%$ CI for continuous outcomes. For pain reduction, at least $30 \%$ difference of VAS scores are needed to be detected after treatment to achieve the minimum clinical therapeutic effect [12]. Thus, we defined minimum, medium, and large SMD effect sizes as $0.3,0.5$, and 0.75 . We used Revman5.2 software from the Cochrane Collaboration for data analyses. Meta-analysis was used if the trials had an acceptable homogeneity on study design, participants, interventions, control, and outcome measures. Statistical heterogeneity was tested by examining $I^{2}$ [13], meaning that an $I$ larger than $50 \%$ indicates the possibility of statistical heterogeneity. Both fixed effect model and random effects model were used if there was possibility of statistical heterogeneity among trials. If $I^{2}$ was less than $50 \%$, only a fixed effect model was used for meta-analysis. Publication bias was explored by funnel plot analysis. Subgroup analyses were conducted to determine the evidence for the different point choice or the different treatment duration if data were sufficient. Sensitivity analyses were in order to determine whether the conclusions were differed if (1) eligibility was restricted to studies without high risk of bias; (2) a fixed effect/random effect model had been applied.

\section{Results}

3.1. Description of Studies. After primary searches in six databases, 1430 citations were identified, as the majority was excluded due to obvious ineligibility, and full text papers of 29 studies were finally retrieved. Finally, 16 randomized trials [14-29] were included in this review (Figure 1), and one trial was published as two separate papers [21, 30] and two unpublished dissertations [31, 32] two trials were reported as dissertations $[24,29]$, one trial [26] was reported in a conference, nine $[14,16,18-20,22,25,27,28]$ of the remaining 13 trials were published in English scientific journals, and other four trials were published in Chinese journals. The characteristics of included trials were listed in Table 1 (Characteristics of included studies). 


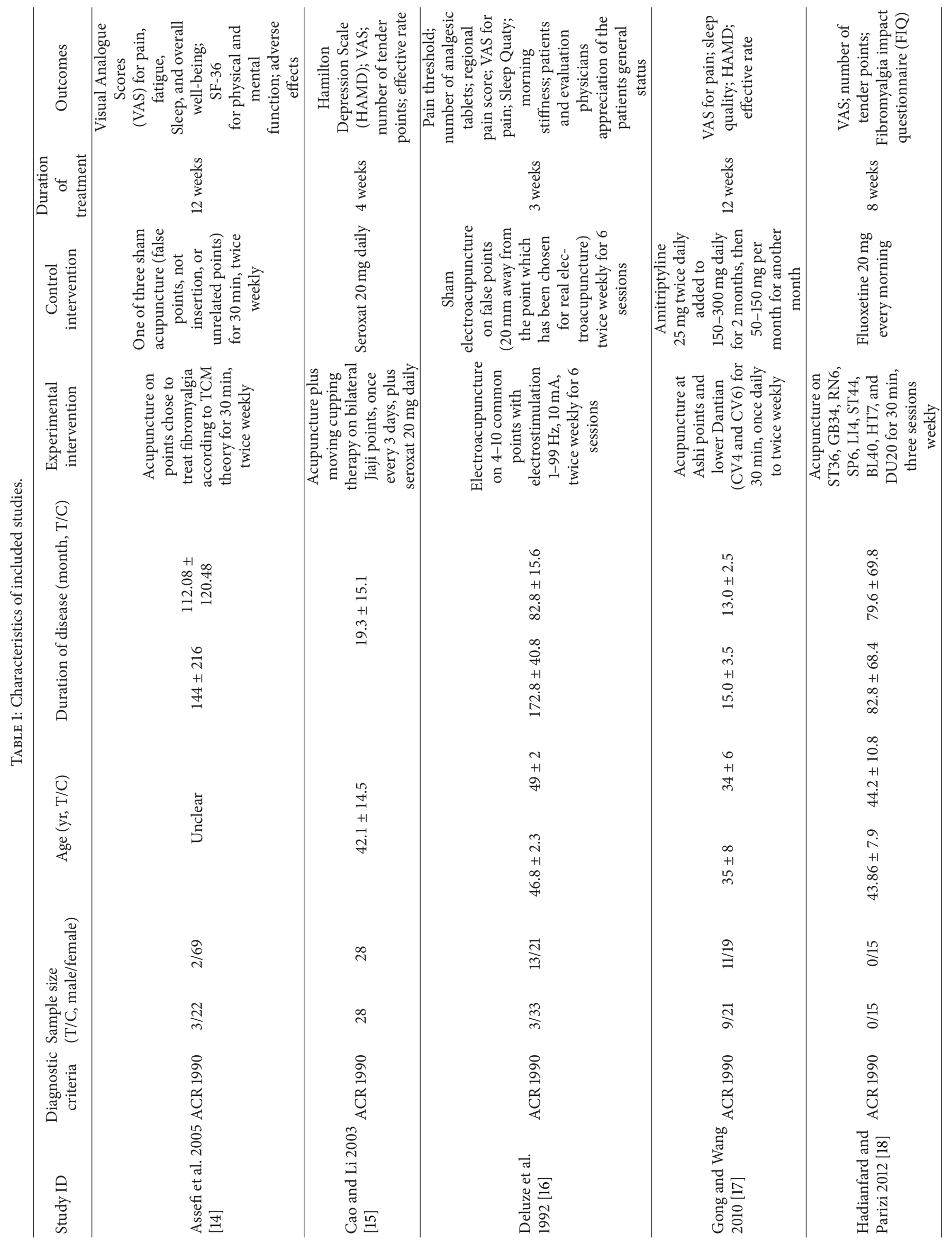




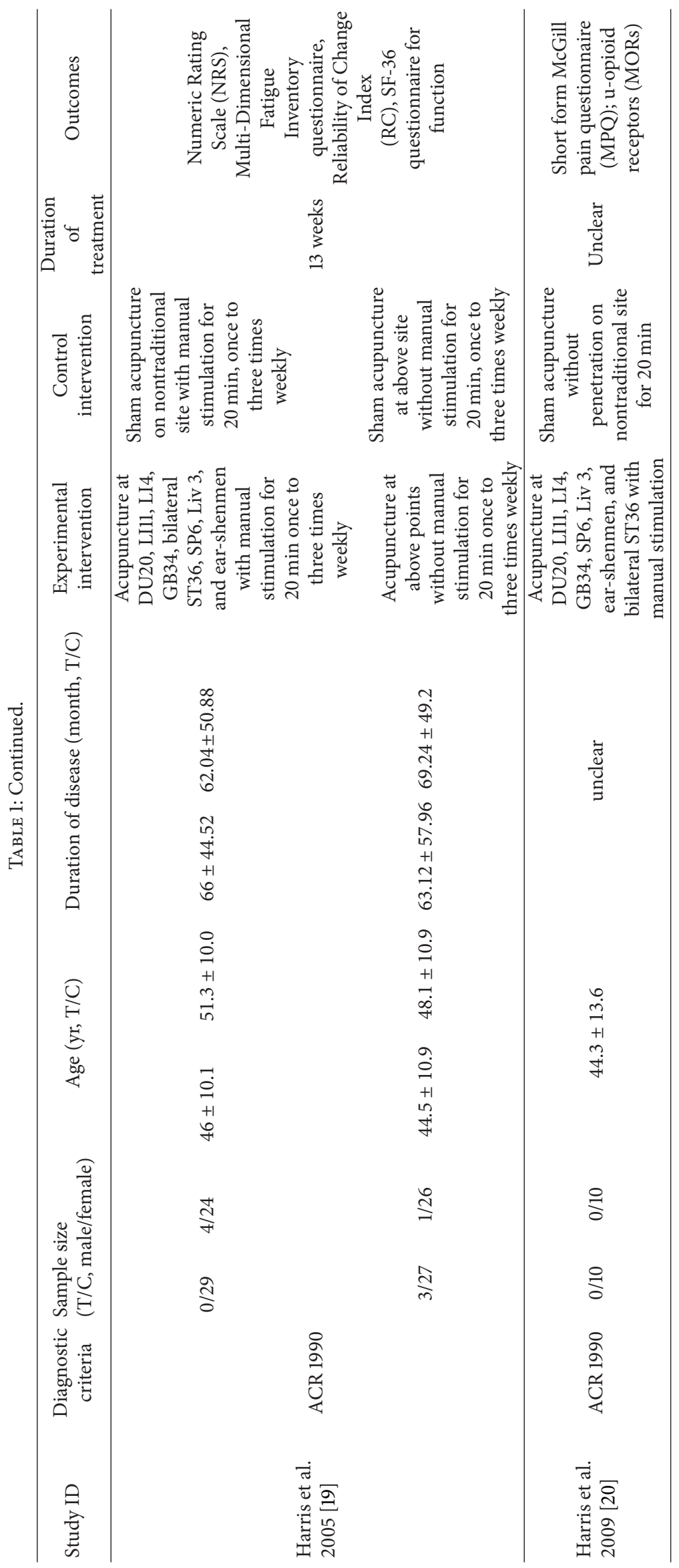




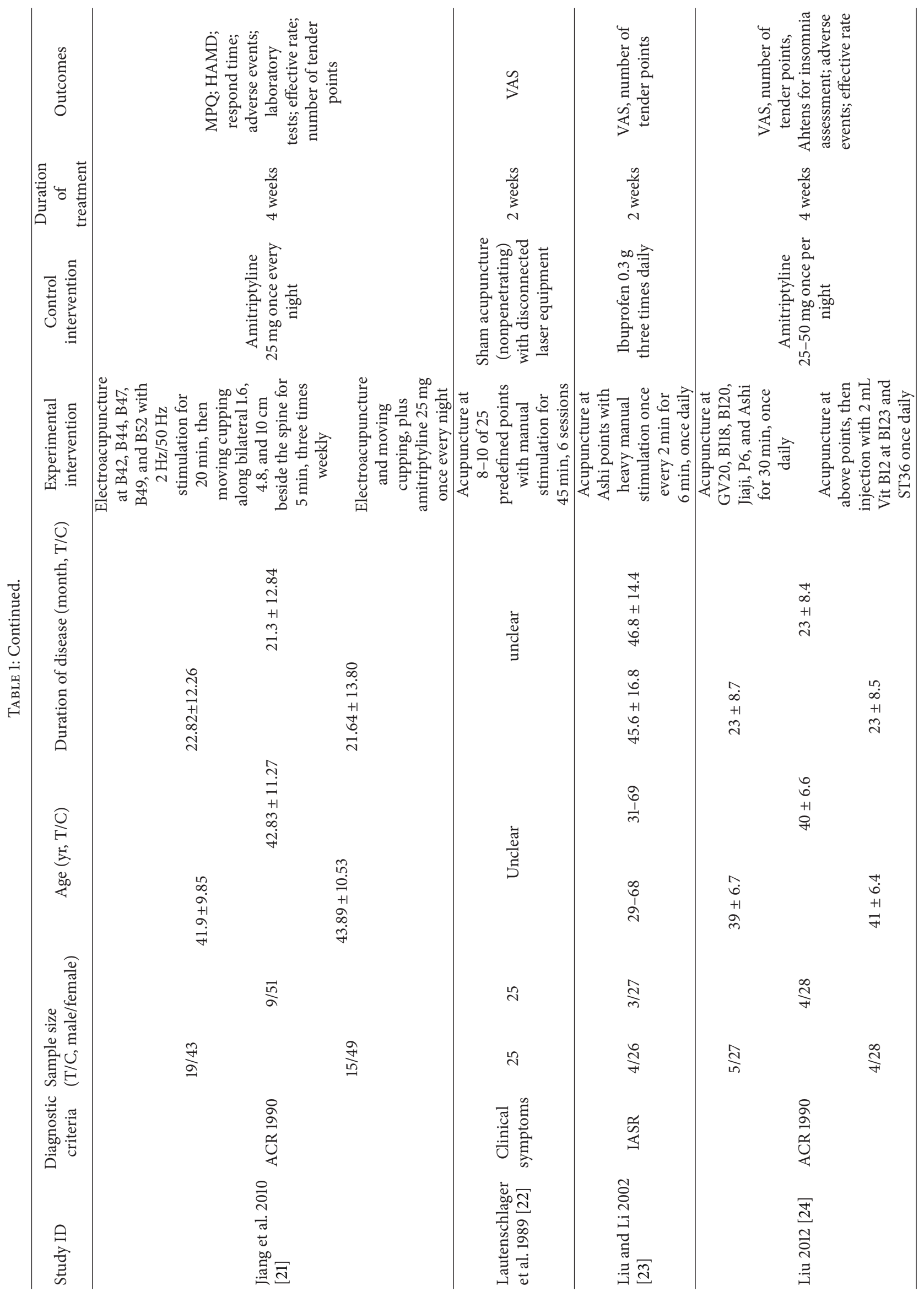




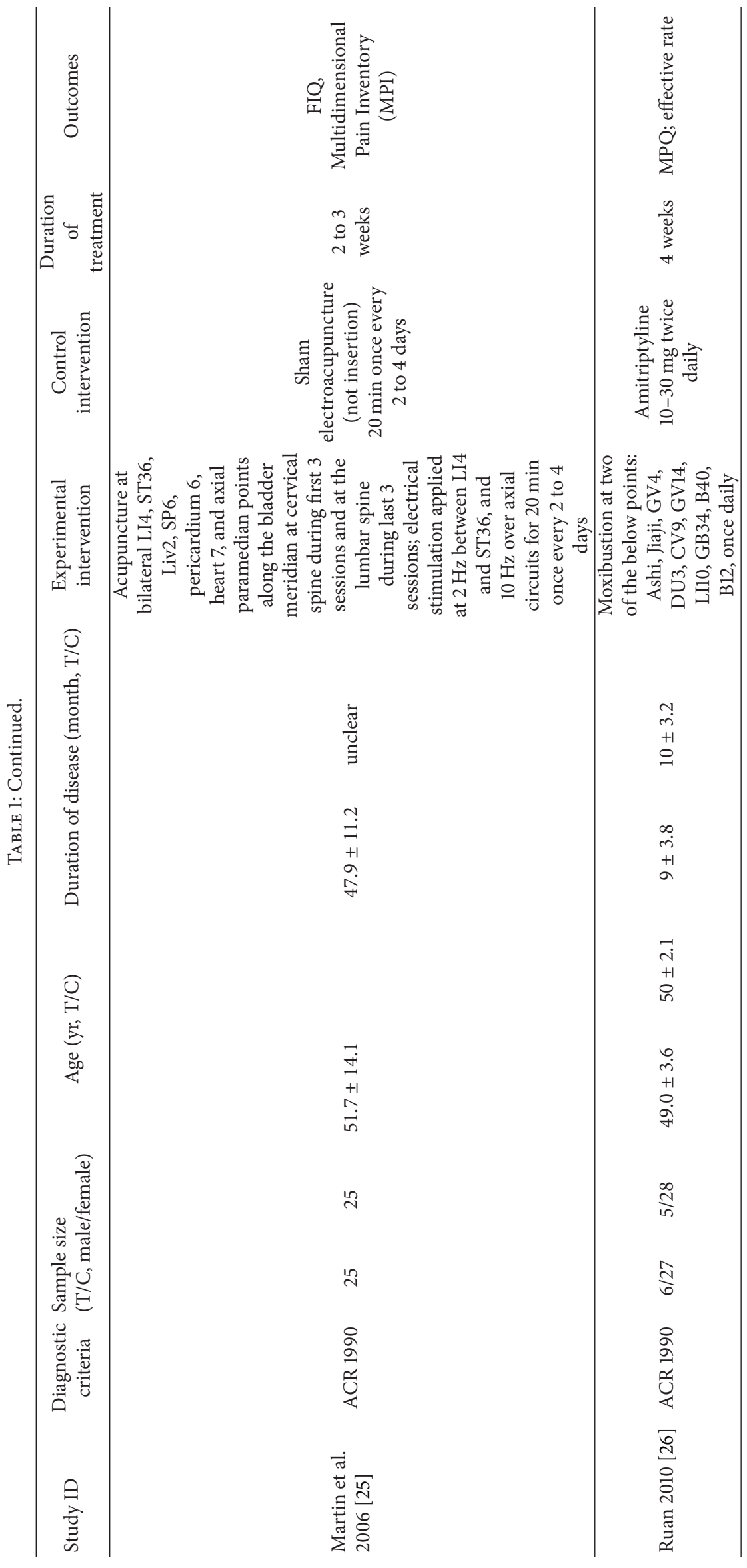




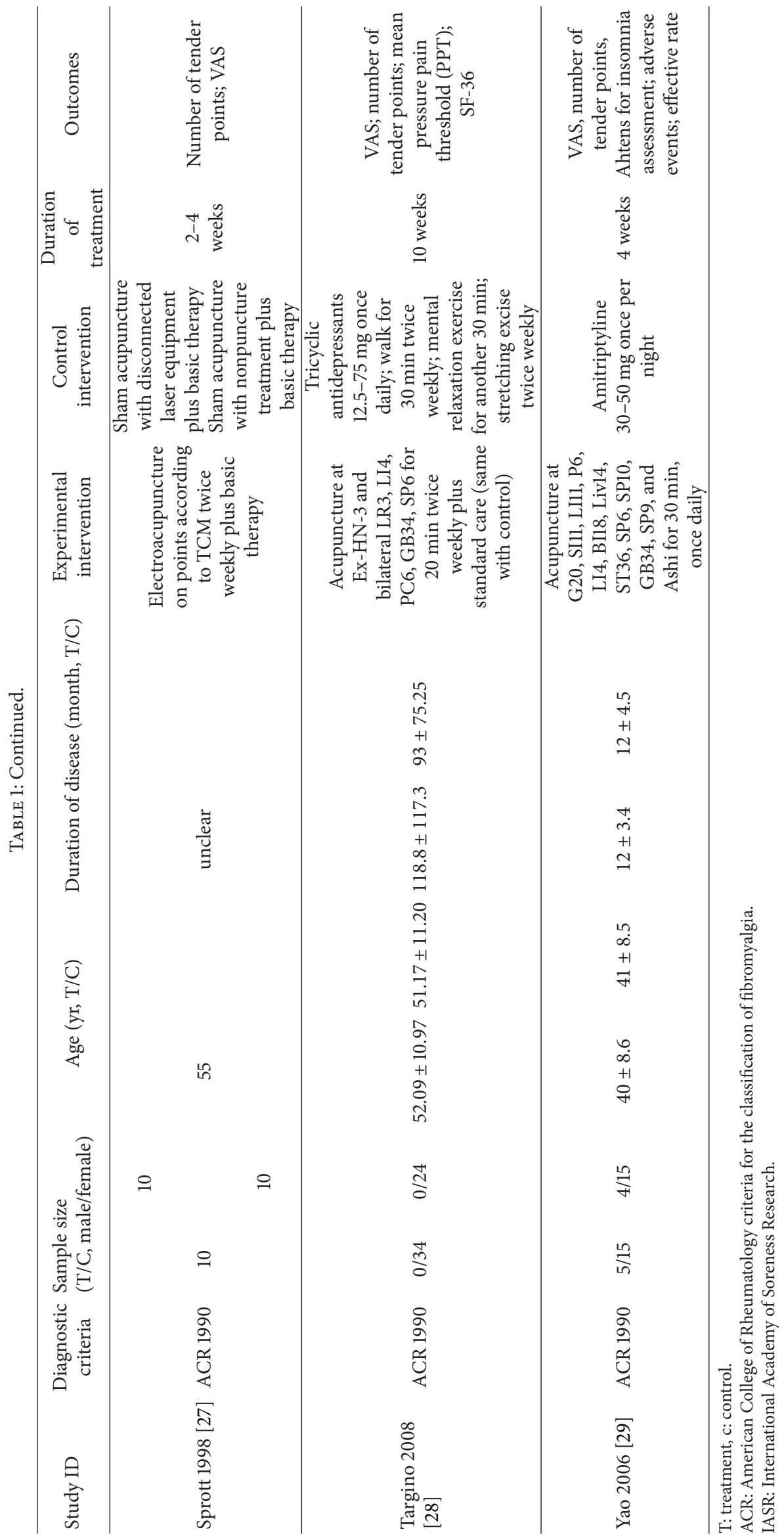




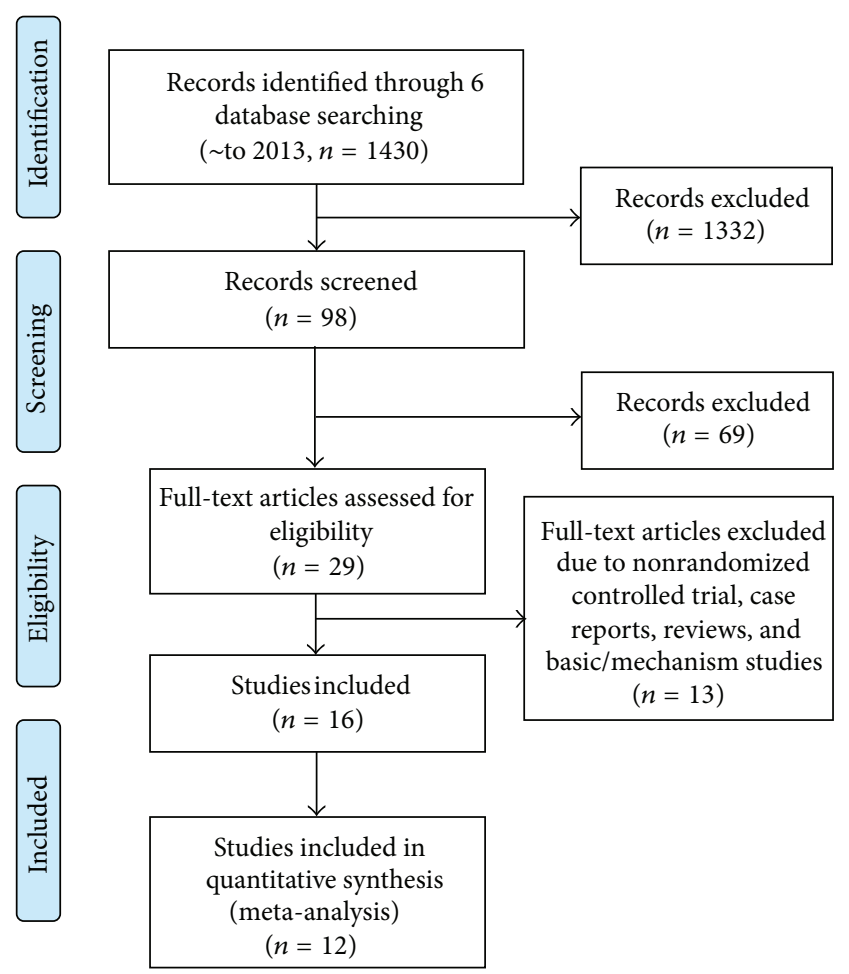

FIgURE 1: Flow chart.

The 16 RCTs involved a total of 1081 patients with FM (an average of 30 patients per group). The participants were aged from 17 to 73 years, and the disease duration was from 4 months to 6 years. All except one trial used ACR 1990 as the diagnostic criteria, and the remaining one trial [22] applied the International Academy of Soreness Research (IASR) [33] for diagnosing FM. The interventions included acupuncture (electroacupuncture, auricular acupuncture), cupping, moxibustion and combinations of acupuncture, and cupping. The controls included no treatment, sham acupuncture or medications. The treatment duration ranged from 2 to 13 weeks. Change of visual analogue scores (VAS) as the major outcome measurement was reported in 11 trials [14-18, 22$24,27,29]$. Eight trials $[15,18,21,23,24,27-29]$ calculated the change of number of tender points, and six trials [18$21,26,27]$ reported results of the McGill Pain Questionnaire (MPQ), Present Pain Intensity (PPI), or Fibromyalgia Impact Questionnaire (FIQ) for assessing intensity of pain. Three trials $[15,17,21]$ used the Hamilton Depression Scale (HAMD) to assess depression. Four trials $[14,16,17,19]$ evaluated quality of life or quality of sleep. Six trials [15, 17, 21, 24, 26, 29] applied four categories to evaluate treatment effects including cure (symptoms disappeared and no tender points exist), markedly effective (symptoms improved more than 50\%), effective/improve (symptoms improved between $25 \%$ and $50 \%$ ), and ineffective (symptoms improved less than $25 \%$ ).

3.2. Methodological Quality. According to our predefined quality assessment criteria, two $[19,20]$ out of the 16 trials (12.5\%) were evaluated as low risk of bias, ten trials were evaluated as high risk of bias, while the other four included

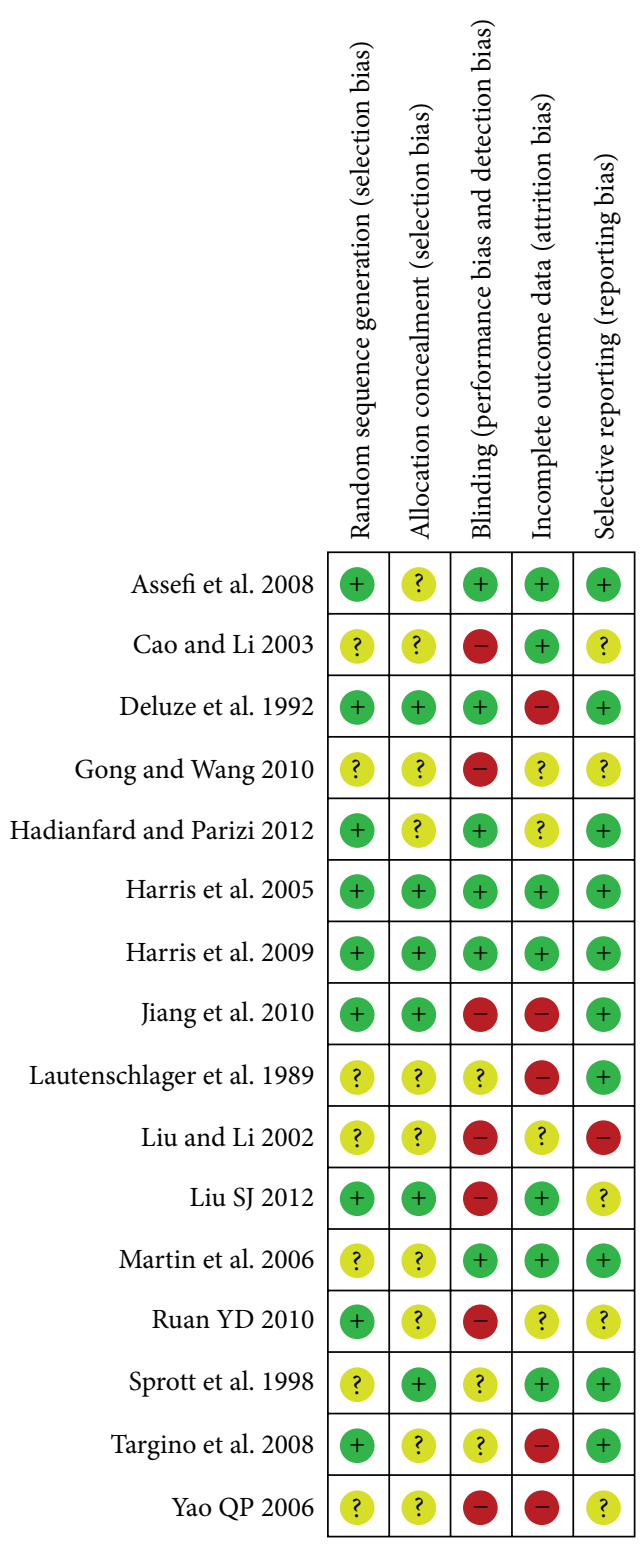

FIGURE 2: Methodological quality summary: review authors' judgments about each methodological quality item for each included study.

trials $[14,18,27,29]$ as unclear risk of bias (Figure 2 Methodological quality summary: review authors' judgments about each methodological quality item for each included study). The sample size varied from 20 to 186 participants, with average of 30 patients per group. Only three trials $[16,18,21]$ reported prior sample size calculation, nine trials $[14,16$, $18-21,24,28]$ described randomization procedures (using random number table or computer generation of random numbers), and six trials [16, 19-21, 24, 27] reported adequate allocation concealment. Three trials $[16,19,20]$ blinded both patients and outcome assessors, four trials [14, 18, 21, 25] blinded only the outcome assessors. Five trials $[16,21,22,28$, 29 ] reported the number of dropouts, and none of these trials used intention-to-treat analysis. 
3.3. Effect Estimates. Thirteen trials [14, 16-20, 25, 27, 29] tested acupuncture for treating FM. Eight trials $[14,16,19,20$, $22,25,27,28]$ compared acupuncture or electroacupuncture with sham acupuncture or sham electroacupuncture, and five trials $[17,18,23,24,29]$ compared acupuncture with medications.

One trial [26] observed the comparison between moxibustion and medication (amitriptyline 10-30 mg daily) for treating FM.

Three trials $[15,21,24]$ tested therapeutic effect of combination therapies of acupoint stimulation for fibromyalgia. Two trials $[15,21]$ compared acupuncture and cupping therapy plus medication with medication only (amitriptyline $25 \mathrm{mg}$ daily), one trial [21] compared acupuncture and cupping therapy with medications, and one trial [24] compared acupuncture plus point injection with amitriptyline (25$50 \mathrm{mg}$ daily).

Due to the insufficient number of the included trials in one meta-analysis, we could not perform a meaningful funnel plot analysis. Results of meta-analysis were listed in Table 2 (estimate effect of included trials in meta-analyses), while results of each individual trials which could not be synthesized in a meta-analyses were concluded in Table 3 (characteristics of randomized controlled trial outside of meta-analysis).

3.4. Therapeutic Effect of Acupoint Stimulation for Pain Relieving. Neither subtotal meta-analysis nor total meta-analysis showed any difference between acupuncture and sham acupuncture on reducing pain (changes between baseline and post-treatment: SMD $-0.09,95 \%$ CI -0.32 to $0.14, I^{2}=2 \%$, random model, $P=0.44,6$ trials; at posttreatment: SMD $-0.22,95 \% \mathrm{CI}-0.51$ to $0.07, I^{2}=26 \%$, random model, $P=$ $0.13,6$ trials). However, one subtotal meta-analysis showed that electroacupuncture was superior to sham electroacupuncture regarding pain reduction after treatment (SMD $-0.42,95 \%$ CI -0.77 to $-0.06, I^{2}=0 \%$, random model, $P=0.02,3$ trials). Two trials $[22,27]$, which could not be included in meta-analysis, also showed no difference between acupuncture and sham acupuncture or no treatment on pain relieve $(P>0.05)$, respectively. The main findings of these trials were presented in Table 3 (characteristics of RCTs outside of meta-analysis). Meta-analysis of five trials $[17,18,23,24,29]$ showed that acupuncture was better than antidepression drugs (amitriptyline $25 \mathrm{mg}$ daily, subtotal: SMD $-0.60,95 \%$ CI -0.93 to $-0.27, I^{2}=22 \%$, random model, $P=0.0004,4$ trials) or the analgesic antipyretic (ibuprofen $0.9 \mathrm{~g}$ daily) with regard to pain reduction according to VAS scores (total: SMD $-0.74,95 \%$ CI -1.13 to $-0.35, I^{2}=55 \%$, random model, $P=0.0002,5$ trials) and the tender points (MD $-2.38,95 \% \mathrm{CI}-3.40$ to $-1.37, I^{2}=0 \%$, fixed model, $P<0.00001,3$ trials).

Two trials $[15,21]$ showed that a combination of acupuncture and cupping therapy plus medications was significantly better than medications (amitriptyline $25 \mathrm{mg}$ daily) alone regarding pain reduction (SMD $-1.65,95 \% \mathrm{CI}-2.10$ to -1.31 , $I^{2}=0 \%$, fixed model, $P<0.00001,2$ trials). However, one trial [21] showed no difference between acupuncture plus cupping therapy and medications (amitriptyline $25 \mathrm{mg}$ daily) for this outcome.

Moxibustion (SMD $-1.46,95 \% \mathrm{CI}-2.00$ to $-0.91, P<$ $0.00001,1$ trials) or combination of acupuncture and point injection (SMD $-1.53,95 \% \mathrm{CI}-2.09$ to $-1.96, P<0.00001,1$ trials) was superior to amitriptyline (10-50 mg daily) regarding pain reduction.

3.5. Therapeutic Effect of Acupoint Stimulation for Improving Depression. No difference between electroacupuncture and sham electroacupuncture was found for improving depression (SMD $-0.33,95 \% \mathrm{CI}-0.90$ to $0.23, P=0.25,1$ trial), which was also true for the combination of acupuncture and cupping therapy (MD $0.90,95 \% \mathrm{CI}-0.68$ to $2.48, P=0.26,1$ trial).

Meta-analysis showed that acupuncture was better than antidepression drugs (amitriptyline or fluoxetine) for improving depression (SMD $-0.67,95 \%$ CI -1.10 to -0.25 , $I^{2}=0 \%$, fixed model, $P=0.02,2$ trials). Two trials also demonstrated that combination of acupuncture and cupping therapy plus medications was better than medications alone for treating FM related depression $(P<0.01)$; however, meta-analysis could not be conducted due to the significant statistical heterogeneity between trials.

3.6. Therapeutic Effect of Acupoint Stimulation for Improving Sleep Quality. Three trials [17, 24, 29] evaluated therapeutic effect of acupuncture for sleep quality after treatment that meta-analysis showed acupuncture was superior to amitriptyline (25-50 mg daily) for improving sleep quality (SMD $-0.32,95 \%$ CI -0.63 to $-0.01, I^{2}=0 \%$, fixed model, $P=0.04$, 3 trials).

One trial [24] showed a significant advantage of combination of acupuncture and point injection on improving sleep quality compared with amitriptyline (25-50 $\mathrm{mg}$ daily, SMD $-0.94,95 \%$ CI -1.46 to $-0.42, P=0.0004,1$ trial).

3.7. Therapeutic Effect of Acupoint Stimulation for Improving FM Related Fatigue. No superior effect of acupuncture was found for fatigue relieve, neither compared to sham acupuncture (SMD $-0.05,95 \%$ CI -0.41 to $0.30, I^{2}=0 \%$, fixed model, $P=0.77,3$ trials) nor compared with anti-depression drugs (fluoxetine $20 \mathrm{mg}$ daily, SMD $-0.27,95 \% \mathrm{CI}-0.99$ to 0.45 , $P=0.46,1$ trial).

3.8. Adverse Events. Only three trials described adverse events $[14,21,29]$, all of which were related to acupuncture or medications. The adverse events of acupuncture included bruising, nausea (3\%), fainting $(0.3-5 \%)$, discomfort at the sites of needle insertion or simulated needle insertion (37\%), and a small amount of bleeding (0.02\%). One trial [14] reported that patients assigned to simulated acupuncture (29\%) had significantly less discomfort than those assigned to real acupuncture (61\%), acupuncture for an unrelated condition (70\%), or sham needling (64\%). Few patients (up to $8.3 \%$ ) with palpitations, fainting, dry mouth, fatigue, and constipation were reported from control medications.

The remaining thirteen trials did not report related information about adverse events during the treatment. 
TABLE 2: Estimated effect sizes of included trials in meta-analyses.

\begin{tabular}{|c|c|c|c|c|}
\hline Trials & Interventions & Estimate effects & $P$ value & $I^{2}$ \\
\hline \multicolumn{5}{|c|}{ (1) Changes of VAS scores for pain } \\
\hline \multicolumn{5}{|c|}{ (1.1) Therapeutic effect of acupuncture } \\
\hline \multicolumn{5}{|c|}{ (1.1.1) Acupuncture versus sham acupuncture } \\
\hline $\begin{array}{l}\text { Assefi et al. } 1989 \\
{[14]}\end{array}$ & $\begin{array}{l}\text { Acupuncture versus sham acupuncture } \\
\text { on false acupoints }\end{array}$ & $0.23[-0.23,0.68]$ & & \\
\hline $\begin{array}{l}\text { Harris et al. } 2005 \\
\text { [19] }\end{array}$ & $\begin{array}{l}\text { Acupuncture on traditional site versus } \\
\text { acupuncture on nontraditional site }\end{array}$ & $0.28[-0.33,0.89]$ & & \\
\hline $\begin{array}{l}\text { Harris et al. } 2005 \\
\text { [19] }\end{array}$ & $\begin{array}{l}\text { Acupuncture on traditional site with } \\
\text { stimulation versus acupuncture on } \\
\text { nontraditional site with stimulation }\end{array}$ & $-0.30[-0.98,0.38]$ & & \\
\hline $\begin{array}{l}\text { Harris et al. } 2009 \\
{[20]}\end{array}$ & $\begin{array}{l}\text { Acupuncture versus sham acupuncture } \\
\text { without penetration on nontraditional } \\
\text { site }\end{array}$ & $-0.14[-1.02,0.74]$ & & \\
\hline \multirow[t]{2}{*}{$\begin{array}{l}\text { Lautenschlager et } \\
\text { al. } 1989 \text { [22] }\end{array}$} & $\begin{array}{l}\text { Acupuncture versus sham acupuncture } \\
\text { with disconnected laser equipment }\end{array}$ & $-0.55[-1.21,0.11]$ & & \\
\hline & Subtotal (random model) & SMD $0.04[-0.37,0.28]$ & 0.79 & $24 \%$ \\
\hline \multicolumn{5}{|c|}{ (1.1.2) Electroacupuncture versus sham electroacupuncture } \\
\hline $\begin{array}{l}\text { Lautenschlager } \\
1989[22]\end{array}$ & $\begin{array}{l}\text { Electroacupuncture versus sham } \\
\text { electroacupuncture on false acupoints }\end{array}$ & $-0.30[-0.84,0.23]$ & & \\
\hline \multirow[t]{3}{*}{$\begin{array}{l}\text { Martin et al. } 2006 \\
{[25]}\end{array}$} & $\begin{array}{l}\text { Electroacupuncture versus sham } \\
\text { electroacupuncture without insertion }\end{array}$ & $-0.12[-0.68,0.44]$ & & \\
\hline & Subtotal (random model) & SMD $-0.22[-0.60,0.17]$ & 0.27 & $0 \%$ \\
\hline & Overall (random model) & SMD $-0.09[-0.32,0.14]$ & 0.44 & $2 \%$ \\
\hline
\end{tabular}

(2) VAS scores for pain after treatment

(2.1) Therapeutic effect of acupuncture

(2.1.1) Acupuncture versus sham acupuncture

(2.1.1.1) Acupuncture versus sham acupuncture

Assefi et al. 1989

[14]

Harris et al. 2005

[19]

Harris et al. 2005

[19]

Harris et al. 2009

[20]

Lautenschlager et al. 1989 [22]

Martin et al. 2006 [25]

Sprott 1998 [27]

Gong and Wang 2010 [17]

Hadianfard and Parizi 2012 [18]
Acupuncture versus sham acupuncture on false acupoints

$0.24[-0.37,0.84]$

Acupuncture on traditional site versus acupuncture on nontraditional site

$0.31[-0.30,0.92]$

Acupuncture on traditional site with stimulation versus acupuncture on nontraditional site with stimulation

Acupuncture versus sham acupuncture without penetration on nontraditional site

Subgroup (random model)

0.75

$43 \%$

(2.1.1.2) Electroacupuncture versus sham electroacupuncture

Electroacupuncture versus sham electroacupuncture on false acupoints

Electroacupuncture versus sham electroacupuncture without insertion

$-0.56[-1.10,-0.02]$

$-0.28[-0.84,0.28]$

Electroacupuncture plus basic therapy

versus sham electroacupuncture with nonpuncture treatment plus basic therapy

Subgroup (random model)

Overall (random model)

(2.1.2) Acupuncture versus medications

$-0.38[-1.27,0.50]$

SMD $-0.42[-0.77,-0.06]$

0.02

$0 \%$

2.1) Acupuncture versus anti-depression drugs

Acupuncture versus amitriptyline

$-0.98[-1.52,-0.44]$

Acupuncture versus fluoxetine

$-0.40[-1.12,0.33]$ 
TABLe 2: Continued.

\begin{tabular}{lccr}
\hline Trials & Interventions & Estimate effects & $P$ value \\
\hline Liu 2012 [24] & Acupuncture versus amitriptyline & $-0.66[-1.16,0.16]$ & $I^{2}$ \\
Yao 2006 [29] & Acupuncture versus amitriptyline & $-0.20[-0.82,0.43]$ & \\
& Subtotal (random model) & SMD -0.60 [-0.93,-0.27] & 0.0004
\end{tabular}

(2.1.2.2) acupuncture versus analgesic-antipyretic

Liu and Li 2002

[23]

$$
-1.34[-1.90,-0.77]
$$

$<0.00001$

NA

Overall (random model)

SMD $-0.74[-1.13,-0.35]$

0.0002

$55 \%$

(2.2) Therapeutic effect of combination of acupuncture and cupping therapy

(2.2.1) Combination of acupuncture and cupping therapy plus medications versus medications alone

Jiang et al. $2010[21]$ Acupuncture plus cupping therapy and seroxat versus seroxat alone

$-1.63[-2.18,-1.08]$

Acupuncture plus cupping therapy and amitriptyline versus amitriptyline

Jiang et al. $2010[21]$ Overall (fixed model)$$
-1.77[-2.74,-0.80]
$$

SMD - 1.65 $[-2.10,-1.31]$

$<0.00001$

0\%

(2.2.2) Combination of acupuncture and cupping therapy versus medications

Jiang et al. 2010 [21]

Acupuncture plus cupping therapy and amitriptyline versus amitriptyline

SMD $-0.21[-0.57,0.15]$

NA

(2.3) therapeutic effect of moxibustion

Ruan 2010 [26]

Moxibustion versus amitriptyline

SMD $-1.46[-2.00,-0.91]$

$<0.00001$

NA

(2.4) Therapeutic effect of combination of acupuncture and point injection

Acupuncture combined with point

Liu 2012 [24] injection (Vit B12) versus amitriptyline

SMD -1.53 [-2.09, -1.96]

$<0.00001$

NA

(3) No. of tender points after treatment

(3.1) Therapeutic effect of acupuncture

(3.1.1) Acupuncture versus medications

(3.1.1.1) Acupuncture versus anti-depression drugs

Liu 2012 [24]

Yao 2006 [29]

Liu and Li 2002

[23]

Jiang et al. 2010 [21]
Acupuncture versus amitriptyline

Acupuncture versus amitriptyline

Subtotal (fixed model)

$$
\begin{aligned}
& -1.50[-3.46,0.46] \\
& -1.70[-4.22,0.82]
\end{aligned}
$$$$
\text { MD }-1.58[-3.12,-0.03]
$$

$0 \%$

(3.1.1.2) acupuncture versus analgesic-antipyretic

$$
\begin{aligned}
& \text { MD -3.00 [-4.35, -1.65] } \\
& \text { MD - } 2.38[-\mathbf{3 . 4 0},-\mathbf{1 . 3 7}]
\end{aligned}
$$$$
<0.0001
$$$$
\text { NA }
$$

Overall (fixed model)

$<0.00001$

0\%

(3.2) Therapeutic effect of combination of acupuncture and cupping therapy

(3.2.1) Combination of acupuncture and cupping therapy versus western medications

Acupuncture plus cupping therapy and amitriptyline versus amitriptyline

$\mathrm{MD}-0.84[-1.72,0.04]$

0.06

NA

(3.2.2) combination of acupuncture and cupping therapy plus western medications versus medications alone

Jiang et al. 2010 [21]

Jiang et al. 2010 [21]

Liu $2012[24]$ seroxat versus seroxat alone

$$
\begin{aligned}
& -3.90[-6.29,-1.51] \\
& -4.70[-5.67,-3.73]
\end{aligned}
$$$$
\text { amitriptyline versus amitriptyline }
$$

Overall (fixed model)

$$
\text { MD -4.59 [-5.49, -3.69] }
$$

$<0.00001$

$\mathbf{0} \%$

(3.3) Therapeutic effect of combination of acupuncture and point injection

Acupuncture combined with point injection (Vit B12) versus amitriptyline

$\mathrm{MD}-1.50[-3.46,0.46]$

0.13

NA

(4) Assessment for depression after treatments

(4.1) Therapeutic effect of acupuncture

(4.1.1) Electroacupuncture versus sham electroacupuncture (FIQ)

Martin et al. 2006

[25]

Gong and Wang 2010 [17]
Electroacupuncture versus sham electroacupuncture without insertion

SMD $-0.33[-0.90,0.23]$

0.25

NA

(4.1.2) acupuncture versus anti-depression drugs

Acupuncture versus amitriptyline (HAMD) 
TABLE 2: Continued.

\begin{tabular}{|c|c|c|c|c|}
\hline Trials & Interventions & Estimate effects & $P$ value & $I^{2}$ \\
\hline \multirow[t]{2}{*}{$\begin{array}{l}\text { Hadianfard and } \\
\text { Parizi } 2012 \text { [18] }\end{array}$} & Acupuncture versus fluoxetine (FIQ) & $-0.48[-1.20,0.25]$ & & \\
\hline & Overall (fixed model) & SMD $-0.67[-1.10,-0.25]$ & 0.02 & $\mathbf{0 \%}$ \\
\hline \multicolumn{5}{|c|}{ (4.2) Therapeutic effect of combination of acupuncture and cupping therapy (HAMD) } \\
\hline \multicolumn{5}{|c|}{ (4.2.1) Combination of acupuncture and cupping therapy versus western medications } \\
\hline Jiang et al. 2010 [21] & $\begin{array}{l}\text { Acupuncture plus cupping therapy and } \\
\text { amitriptyline versus amitriptyline }\end{array}$ & MD $0.90[-0.68,2.48]$ & 0.26 & NA \\
\hline \multicolumn{5}{|c|}{ (4.2.2) combination of acupuncture and cupping therapy plus medications versus medications alone } \\
\hline $\begin{array}{l}\text { Cao and Li } 2003 \\
{[15]}\end{array}$ & $\begin{array}{l}\text { Acupuncture plus cupping therapy and } \\
\text { seroxat versus seroxat alone }\end{array}$ & $\mathrm{MD}-6.00[-8.36,-3.64]$ & $<0.00001$ & NA \\
\hline Jiang et al. 2010 [21] & $\begin{array}{l}\text { Acupuncture plus cupping therapy and } \\
\text { amitriptyline versus amitriptyline }\end{array}$ & $\mathrm{MD}-1.78[-2.97,-0.59]$ & 0.003 & NA \\
\hline
\end{tabular}

(5) Assessment for sleep quality after treatments

(5.1) therapeutic effect of acupuncture

Gong and Wang 2010 [17]

Liu 2012 [24]

Yao 2006 [29]

Liu $2012[24]$
Acupuncture versus amitriptyline

Acupuncture versus amitriptyline

Acupuncture versus amitriptyline

$$
\begin{aligned}
& -0.34[-0.85,0.17] \\
& -0.11[-0.74,0.52] \\
& -0.43[-0.93,0.07]
\end{aligned}
$$

SMD $-0.32[-0.63,-0.01] \quad 0.04 \quad 0 \%$

(5.2) Therapeutic effect of combination of acupuncture and point injection

Acupuncture combined with point injection (Vit B12) versus amitriptyline

(6) FQI after treatments

(6.1) Therapeutic effect of acupuncture

(6.1.1) Electroacupuncture versus sham electroacupuncture

Martin et al. 2006

[25]

Electroacupuncture versus sham electroacupuncture without insertion

$$
\mathrm{MD}-4.30[-11.08,2.48]
$$

0.21

NA

(6.1.2) Acupuncture versus antidepression drugs

Hadianfard and

Parizi 2012 [18]

Acupuncture versus fluoxetine

$\mathrm{MD}-4.60[-12.42,3.22]$

0.25

NA

(7) Assessment for fatigue after treatments

(7.1) Therapeutic effect of acupuncture

(7.1.1) Acupuncture versus sham acupuncture

(7.1.1.1) Electroacupuncture versus sham electroacupuncture

Martin et al. 2006

[25]

Harris et al. 2005

[19]

Harris et al. 2005

[19]
SMD $-0.23[-0.79,0.33]$

0.42

NA

$0.11[-0.50,0.71]$

$0.01[-0.67,0.68]$
Electroacupuncture versus sham electroacupuncture without insertion

(7.1.1.2) acupuncture versus sham acupuncture

Acupuncture on traditional site versus acupuncture on nontraditional site

Acupuncture on traditional site with stimulation versus acupuncture on nontraditional site with stimulation

Subtotal (fixed model)

Overall (fixed model)
0.79

0.77

SMD -0.05 [-0.41, 0.30]

$0 \%$

0\%

\section{(7.1.2) Acupuncture versus antidepression drugs}

Hadianfard and Acupuncture versus fluoxetine

SMD -0.27 [-0.99, 0.45]

0.46

NA

MD: mean difference.

TCM: traditional Chinese medicine.

TENS: transcutaneous electrical nerve stimulation.

RR: risk ratio. 
TABLE 3: Characteristic of randomized controlled trials outside meta-analysis.

\begin{tabular}{|c|c|c|}
\hline Study ID & Comparisons & Main findings \\
\hline \multicolumn{3}{|c|}{ Acupuncture versus sham acupuncture } \\
\hline $\begin{array}{l}\text { Lautenschlager } \\
1989[22]\end{array}$ & $\begin{array}{c}\text { Acupuncture versus } \\
\text { sham laser acupuncture }\end{array}$ & $\begin{array}{l}\text { There was significant difference between } \\
\text { acupuncture and sham treatment in pain } \\
\text { reduction measured for all } 3 \text { methods by } \\
\text { end of treatment. At follow up of } 3 \text { months } \\
\text { after the last treatment, no significant } \\
\text { changes were observed }(P>0.05) \text {. }\end{array}$ \\
\hline Sprott 1998 [27] & $\begin{array}{c}\text { Acupuncture versus } \\
\text { sham laser acupuncture }\end{array}$ & $\begin{array}{l}\text { The data for pain reduction by tender points } \\
\text { were not completely reported, but the results } \\
\text { showed that the number of tender points } \\
\text { was not significantly decreased after } \\
\text { acupuncture treatment in comparison to } \\
\text { sham treatment }(P>0.05) \text {. The intensity of } \\
\text { pain, measured by the VAS, also showed no } \\
\text { significant reduction, neither immediately at } \\
\text { the end of treatment or } 2 \text { months after the } \\
\text { treatment }(P>0.05) \text {. }\end{array}$ \\
\hline
\end{tabular}

Acupuncture versus no treatment

Sprott 1998 [27] Acupuncture versus no

The number of tender points was significantly decreased after acupuncture treatment in comparison to no treatment

$(P>0.05)$.

Acupuncture plus standard cares versus standard cares alone

Acupuncture plus

tricyclic antidepressants

and exercise with

Targino 2008 [28] tricyclic antidepressants and exercise
Patients in acupuncture group were significantly better than the control group in terms of VAS scores $(P<0.001)$, pressure pain threshold (PPT) $(P<0.001)$, the number of tender points below $4 \mathrm{~kg} / \mathrm{cm}^{2}(P<0.001)$, and in 5 subscales of the SF-36 $(P<0.05)$.
No serious adverse event was reported in all included trials.

\section{Discussions}

Our review with 16 included trials demonstrates that acupuncture or combination of acupuncture and cupping therapy was significantly more effective than conventional medications (anti-depression drugs or analgesic antipyretic) on reducing pain and improving the FM related symptoms (such as depression or fatigue), and however, the therapeutic effects of other types of acupoint stimulation are uncertain due to the limited numbers of clinical trials.

There are several limitations of this review. The quality of the included studies is generally poor, which indicates high or unclear risk of bias due to insufficient reporting of methodological components from the trials. There was unclear description of randomization procedure and lack of blinding in half of the trials which may create potential performance bias and detection bias as patients and researchers might be aware of the therapeutic interventions. Intentionto-treat analysis was not applied in most of the included trials. We were limited in our ability to perform meta-analysis due to the incomplete outcome data reporting in three included trials $[14,25,28]$. Outcomes such as cure, markedly effective, effective, or ineffective were used in six trials, these are not validated, and the finding will be hard to interpret. Consequently, the interpretation of these positive findings needs to be cautious, and the study methodology needs to be improved for future confirmatory studies.

Though we could not perform a meaningful funnel plot due to the insufficient number of included trials in meta-analysis, there was potential publication bias among included trials. All the nine English publications reported no significant statistical difference between groups, but five out of seven Chinese publications showed significant advantages of acupoint stimulation compared with control. One of the potential reasons is all but one trial [18] conducted outside China employed the comparison between acupuncture and sham acupuncture, but sham acupuncture may not be appropriate as a placebo against which to evaluate the therapeutic effect of real acupuncture [34]. Due to the sociocultural background, Chinese participants may have a preference for acupuncture treatment compared to a pharmacological intervention, which may create potential performance bias and affect the results of assessment with patients report outcome. Besides, no trial used syndrome differentiation for acupuncture point selection.

Comparing to previous systematic reviews [6-8] which assessed acupuncture, our review included two more English 
publications and four more trials conducted in China. Results of the comparison between acupuncture and sham acupuncture were similar to other reviews due to the same data source; however, regardless of the poor methodological quality of included trials, we found that acupuncture compared to medications showed significant therapeutic effects on improving FM related symptoms (including pain, fatigue, depression, or insomnia). We also assessed trials focusing on the effect of other acupoint stimulation, but no confirmed conclusions could be drawn due to the insufficient number of relevant trials.

Considering the small sample sizes and unclear or high risk of bias of the current included trials, we suggest that trials with rigorous designing should be conducted to further confirm the effectiveness of acupoint stimulation in treating FM. Although there is not ideal placebo for acupoint stimulation and blinding of the patients and practitioners might be very difficult for acupuncture or herbal medicine, blinding of outcome assessors should be attempted as far as possible to minimize performance and assessment biases. Choosing outcome measures should be based on international consensus and include continuous data and daily average pain scores from baseline to study completion. Using appropriate methods (such as intention to treat analysis) to deal with missing data is vital as is the application of welldefined diagnostic criteria, such as ACR 2009. Reporting of trials should follow the Consolidated Standards of Reporting Trials (CONSORT) [35] or Standards for Reporting Interventions in Clinical Trials of Acupuncture (STRICTA) [36] to explicitly explain the processes involved transparently. Our preliminary conclusions suggest that patients with FM might benefit from acupoint stimulation, especially acupuncture or combination of acupuncture and cupping therapy. However, more high quality clinical evidence is needed to further testify this conclusion.

4.1. Key Message. There is low level evidence showing that acupuncture or a combination of acupuncture and cupping therapy was significantly more effective than conventional medications (antidepression drugs or analgesic antipyretic) on reducing pain and improving the FM related symptoms (such as depression or fatigue).

Acupuncture seems to have no better effect than sham acupuncture with regard to pain relieve in patients with fibromyalgia according to the current moderate level clinical evidence.

The therapeutic effects of other types of acupoint stimulation are uncertain due to limited numbers of clinical trials.

\section{Conflict of Interests}

The authors declare that there is no conflict of interests.

\section{Funding}

Huijuan Cao and Jianping Liu were supported by the Research Capacity Establishment Grant (nos. 201207007 and 0100604024) of Beijing University of Chinese Medicine. This work was also supported by the Grant no. 2011-CXTD-09.

\section{References}

[1] I. J. Russell and K. G. Raphael, "Fibromyalgia syndrome: presentation, diagnosis, differential diagnosis, and vulnerability," CNS Spectrums, vol. 13, no. 3, supplement 5, pp. 6-11, 2008.

[2] F. Wolfe, H. A. Smythe, M. B. Yunus et al., "The American college of rheumatology 1990. Criteria for the classification of fibromyalgia. Report of the multicenter criteria committee," Arthritis and Rheumatism, vol. 33, no. 2, pp. 160-172, 1990.

[3] F. Wolfe, D. J. Clauw, M. Fitzcharles et al., "The American college of rheumatology preliminary diagnostic criteria for fibromyalgia and measurement of symptom severity," Arthritis Care and Research, vol. 62, no. 5, pp. 600-610, 2010.

[4] C. E. Argoff, "Pharmacologic management of chronic pain," Journal of the American Osteopathic Association, vol. 102, no. 9, supplement 3, pp. 521-526, 2002.

[5] E. Mayhew and E. Ernst, "Acupuncture for fibromyalgia-a systematic review of randomized clinical trials," Rheumatology, vol. 46, no. 5, pp. 801-804, 2007.

[6] E. Martin-Sanchez, E. Torralba, E. Díaz-Domínguez, A. Barriga, and J. L. R. Martin, "Efficacy of acupuncture for the treatment of fibromyalgia: systematic review and meta-analysis of randomized trials," Open Rheumatology Journal, vol. 3, pp. 25-29, 2009.

[7] J. Langhorst, P. Klose, F. Musial, D. Irnich, and W. Häuser, "Efficacy of acupuncture in fibromyalgia syndrome-a systematic review with a meta-analysis of controlled clinical trials," Rheumatology, vol. 49, no. 4, pp. 778-788, 2010.

[8] J. C. Deare, Z. Zheng, C. C. Xue et al., "Acupuncture for treating fibromyalgia," Cochrane Database of Systematic Reviews, no. 5, Article ID CD007070, 2013.

[9] H. Cao, J. Liu, and G. T. Lewith, "Traditional Chinese medicine for treatment of fibromyalgia: a systematic review of randomized controlled trials," Journal of Alternative and Complementary Medicine, vol. 16, no. 4, pp. 397-409, 2010.

[10] X. Y. Fu, Y. Li, and J. J. Yang, "A survey of acupuncture for fibromyalgia syndrome," Shanghai Journal of Acupuncture and Moxibustion, vol. 23, no. 7, pp. 46-48, 2004.

[11] J. P. T. Higgins and S. Green, Eds., Cochrane Handbook for Systematic Reviews of Interventions Version 5.1.0, The Cochrane Collaboration, 2011, http://www.cochrane-handbook.org.

[12] A. M. Kelly, "Does the clinically significant difference in visual analog scale pain scores vary with gender, age, or cause of pain?" Academic Emergency Medicine, vol. 5, no. 11, pp. 1086-1090, 1998.

[13] J. P. T. Higgins and S. G. Thompson, "Quantifying heterogeneity in a meta-analysis," Statistics in Medicine, vol. 21, no. 11, pp. 15391558, 2002.

[14] N. P. Assefi, K. J. Sherman, C. Jacobsen, J. Goldberg, W. R. Smith, and D. Buchwald, "A randomized clinical trial of acupuncture compared with sham acupuncture in fibromyalgia," Journal of Rehabilitation Medicine, vol. 40, no. 7, pp. 582-588, 2008.

[15] J. Q. Cao and Y. Li, "Combination of acupuncture and antidepressant medications in treating of 56 cases of fibromyalgia," Chinese Archives of Traditional Chinese Medicine, vol. 21, no. 5, pp. 813-817, 2003.

[16] C. Deluze, L. Bosia, A. Zirbs, A. Chantraine, and T. L. Vischer, "Electroacupuncture in fibromyalgia: results of a controlled trial," The British Medical Journal, vol. 305, no. 6864, pp. 1249$1252,1992$.

[17] W. Z. Gong and Y. Q. Wang, "Observations on the therapeutic effect of acupuncture on fibromyalgia syndrome," Shanghai 
Journal of Traditional Chinese Medicine, vol. 29, no. 11, pp. 725727, 2010.

[18] M. J. Hadianfard and M. H. Parizi, "A randomized clinical trial of fibromyalgia treatment with acupuncture compared with fluoxetine," Iranian Red Crescent Medical Journal, vol. 14, no. 10, pp. 631-640, 2012.

[19] R. E. Harris, X. Tian, D. A. Williams et al., "Treatment of fibromyalgia with formula acupuncture: investigation of needle placement, needle stimulation, and treatment frequency," Journal of Alternative and Complementary Medicine, vol. 11, no. 4, pp. 663-671, 2005.

[20] R. E. Harris, J. K. Zubieta, D. J. Scott, V. Napadow, R. H. Gracely, and D. J. Clauw, "Traditional Chinese acupuncture and placebo (sham) acupuncture are differentiated by their effects on $\mu$ opioid receptors (MORs)," NeuroImage, vol. 47, no. 3, pp. 10771085, 2009.

[21] Z. Y. Jiang, C. D. Li, L. Qiu et al., "Combination of acupuncture, cupping and medicine for treatment of fibromyalgia syndrome: a multi-central randomized controlled trial," Chinese Acupuncture and Moxibustion, vol. 30, no. 4, pp. 265-269, 2010.

[22] J. Lautenschlager, C. C. Schnorrenberger, and W. Muller, "Acupuncture in the treatment of generalized tendomyopathy (fibromyalgia syndrome)," Deutsche Zeitschrift fur Akupunktur, vol. 32 , no. 6 , pp. $122-128,1989$.

[23] Q. Liu and F. Li, "Clinical observation of acupuncture for 30 cases of fibromyalgia," Anthology of Medicine, vol. 21, no. 2, article 183, 2002.

[24] S. J. Liu, Clinical study on treating fibromyalgia syndrome with acupuncture and point injection [M.S. thesis], Hubei University of Traditional Chinese Medicine, 2012.

[25] D. P. Martin, C. D. Sletten, B. A. Williams, and I. H. Berger, "Improvement in fibromyalgia symptoms with acupuncture: results of a randomized controlled trial," Mayo Clinic Proceedings, vol. 81, no. 6, pp. 749-757, 2006.

[26] Y. D. Ruan, W. Z. Wei, X. T. Hong, and X. H. Yang, "Clinical observation of moxibustion for fibromyalgia," in Proceedings of the 11th Acupuncture Symposium of Guangdong Province, 2010.

[27] H. Sprott, "Efficiency of acupuncture in patients with fibromyalgia," Clinical Bulletin of Myofascial Therapy, vol. 3, no. 1, pp. 3743, 1998.

[28] R. A. Targino, M. Imamura, H. H. S. Kaziyama et al., "A randomized controlled trial of acupuncture added to usual treatment for fbromyalgia," Journal of Rehabilitation Medicine, vol. 40, no. 7, pp. 582-588, 2008.

[29] Q. P. Yao, Clinical study on fibromyalgia syndrome with the acupuncture of stimulating Du channel and smoothing other channels and collaterals [M.S. thesis], Heilongjiang University of Traditional Chinese Medicine, 2006.

[30] C. D. Li, X. Y. Fu, Z. Y. Jiang et al., "Clinical study on combination of acupuncture, cupping and medicine for treatment of fibromyalgia syndrome," Chinese Acupuncture and Moxibustion, vol. 26, no. 1, pp. 8-10, 2006.

[31] X. Y. Fu, Clinical randomized controlled trial on combination of acupuncture, cupping, and medicine for treatment of fibromyalgia syndrome [M.S. thesis], Chengdu University of Traditional Chinese Medicine, 2004.

[32] Y. Yue, The clinical study of combination therapy with acupuncture, cupping and medicine for fibromyalgia syndrome [M.S. thesis], Chengdu University of Traditional Chinese Medicine, 2010.

[33] K. Ronald, Pain Management Secrets, China Ocean Press, Beijing, China, 1999.
[34] M. V. Madsen, P. C. Gøtzsche, and A. Hróbjartsson, "Acupuncture treatment for pain: systematic review of randomised clinical trials with acupuncture, placebo acupuncture, and no acupuncture groups," The British Medical Journal, vol. 338, Article ID a3115, 2009.

[35] K. F. Schulz, D. G. Altman, D. Moher, and The CONSORT Group, "CONSORT 2010 statement: updated guidelines for reporting parallel group randomised trials," The British Medical Journal, vol. 340, article c332, 2010.

[36] H. MacPherson, D. G. Altman, R. Hammerschlag et al., "Revised standards for reporting interventions in clinical trials of acupuncture (STRICTA): extending the CONSORT statement," PLoS Medicine, vol. 7, no. 6, Article ID e1000261, 2010. 


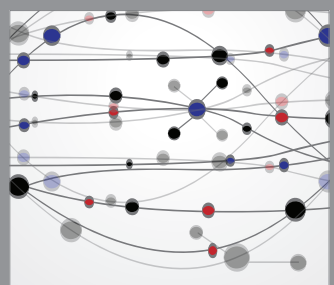

The Scientific World Journal
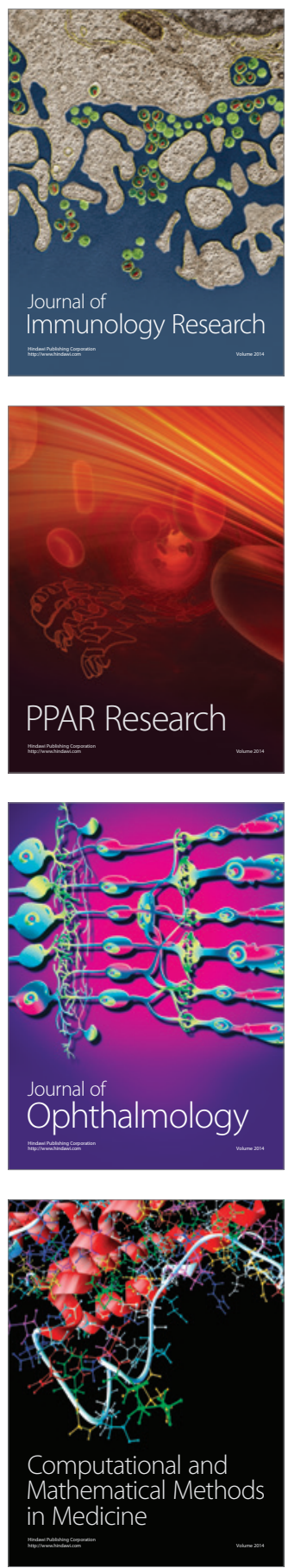

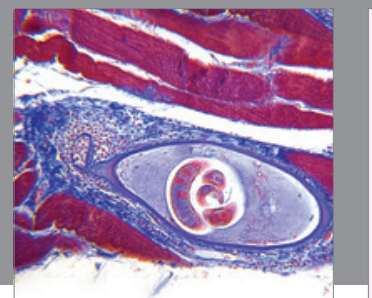

Gastroenterology

Research and Practice
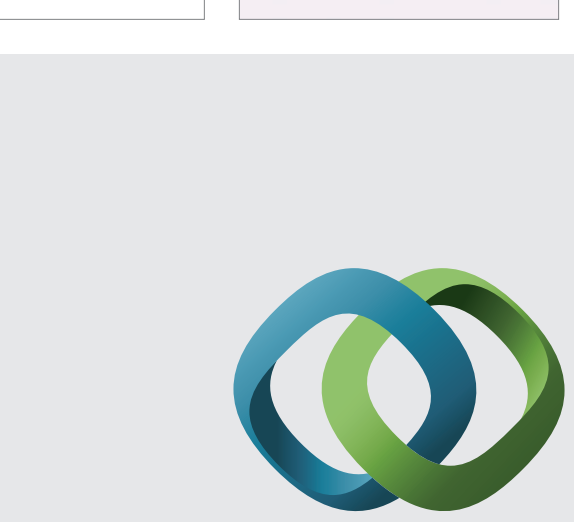

\section{Hindawi}

Submit your manuscripts at

http://www.hindawi.com
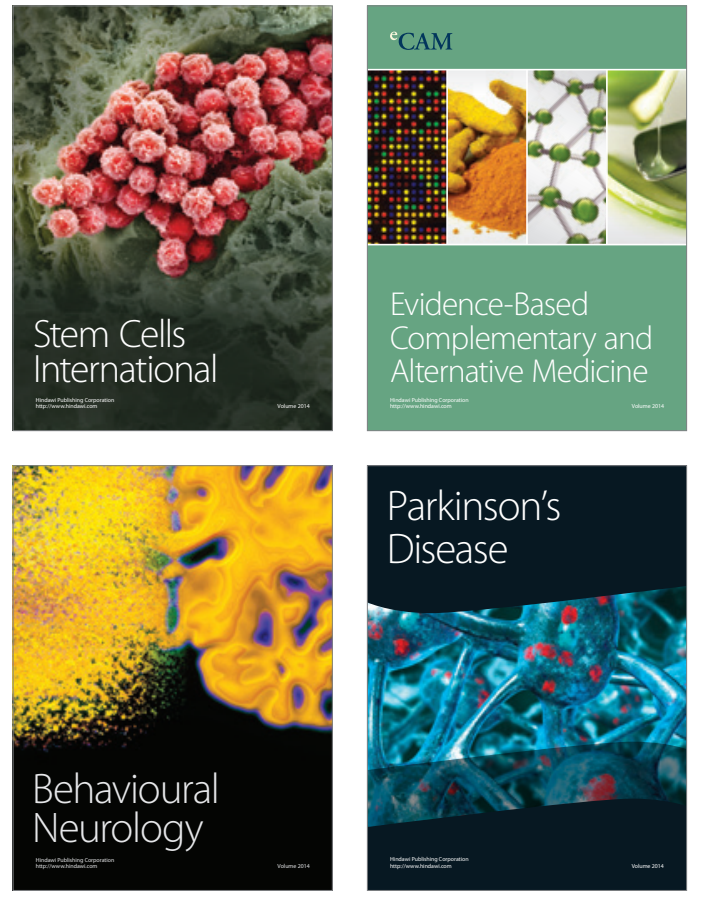
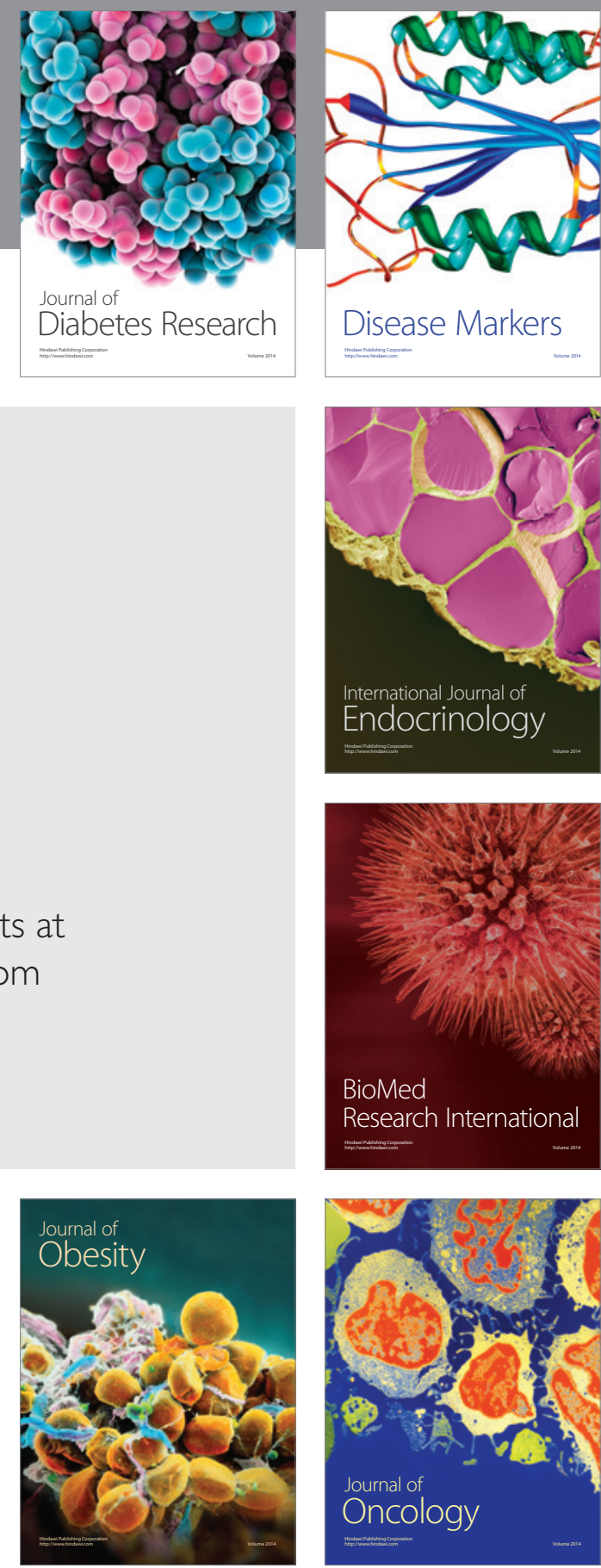

Disease Markers
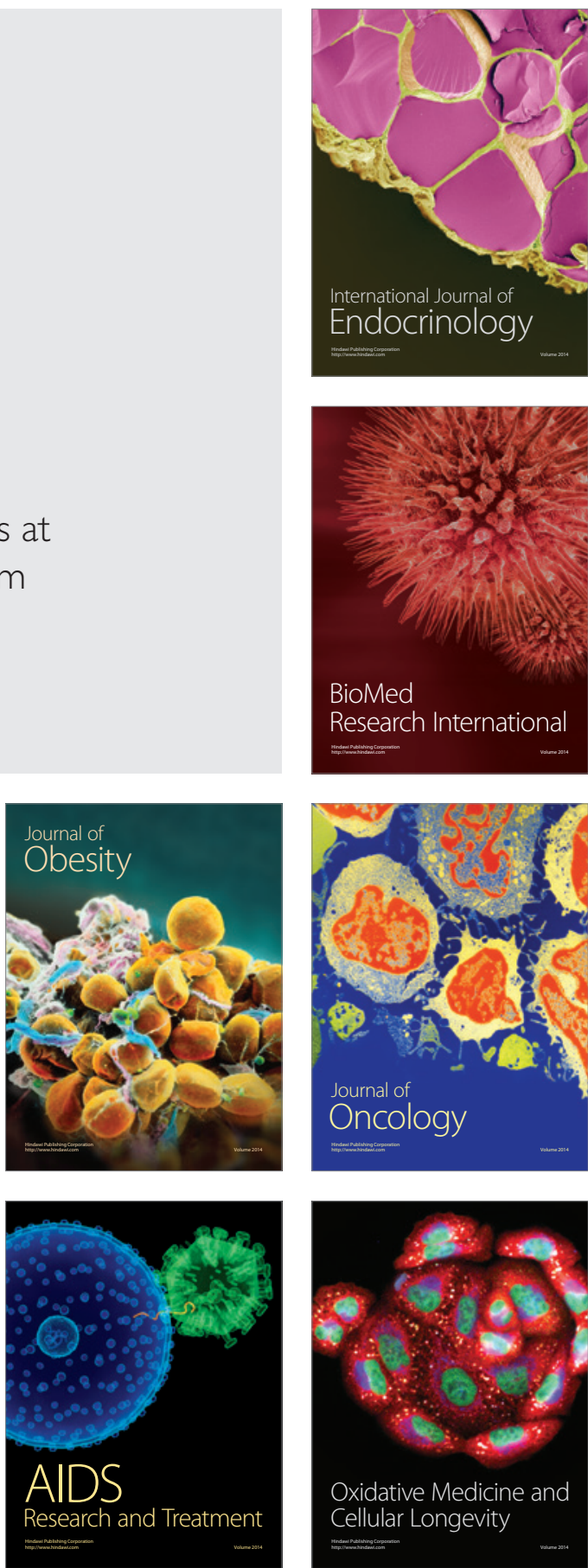